
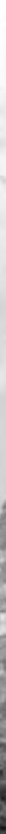

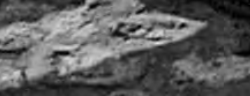

ect 12

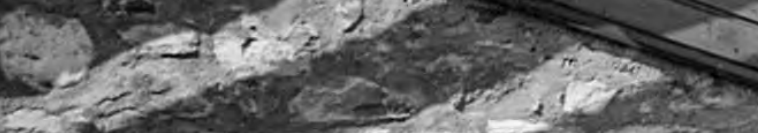

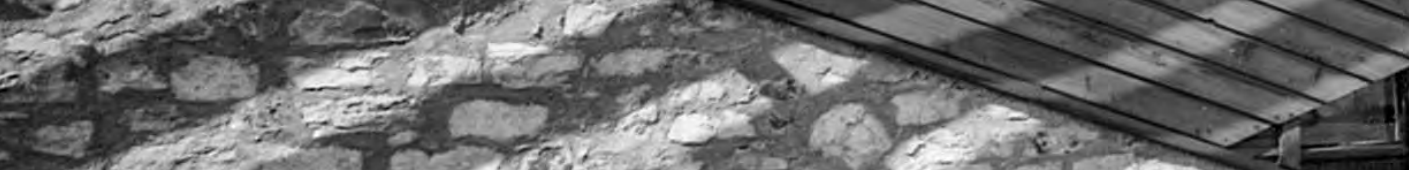

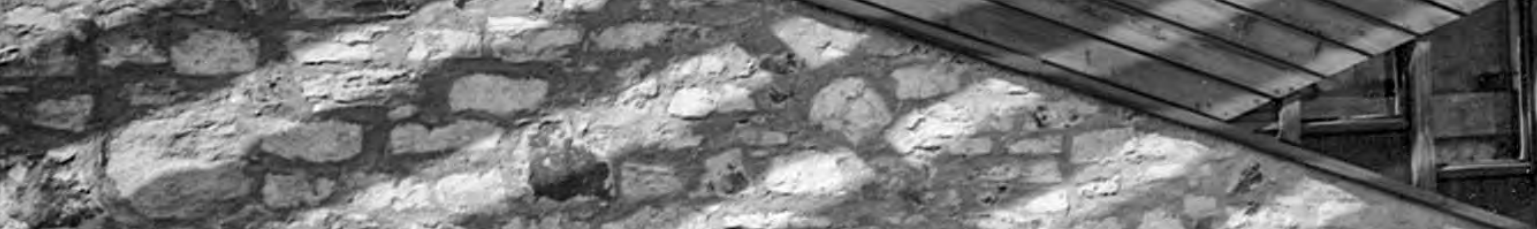
-

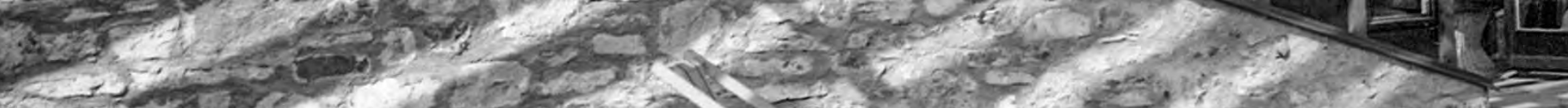

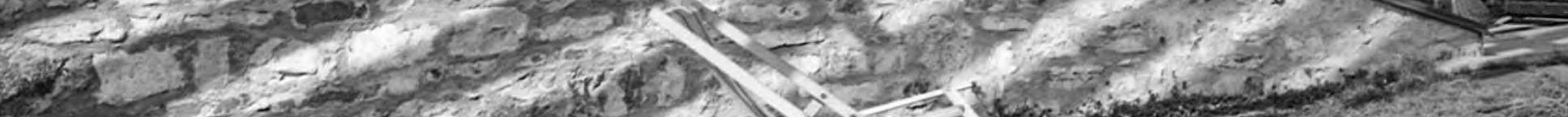
-

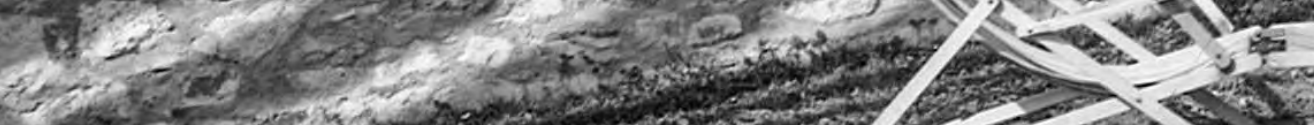

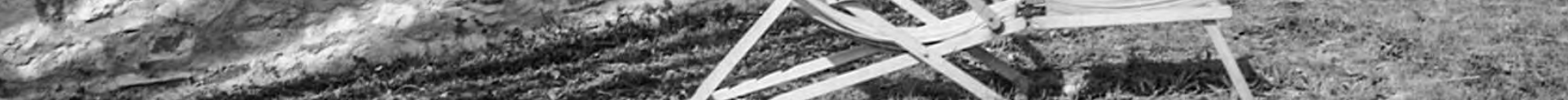
rem thest

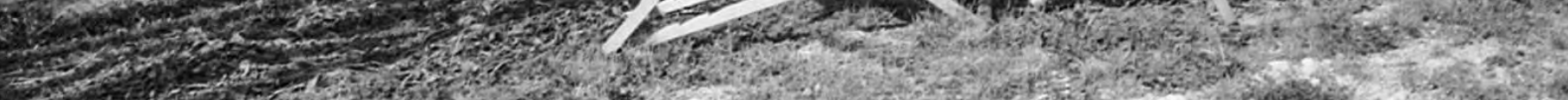

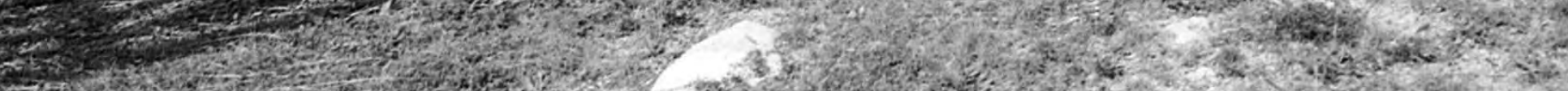

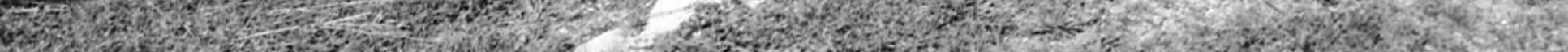

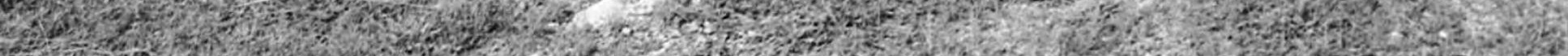

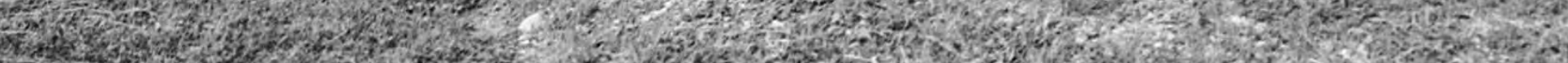

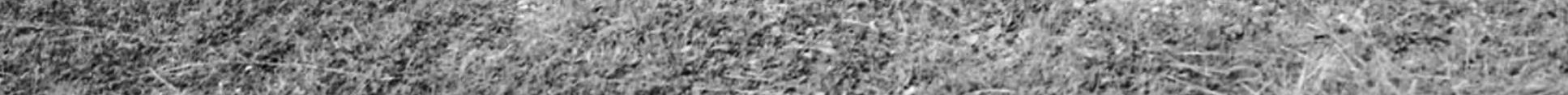

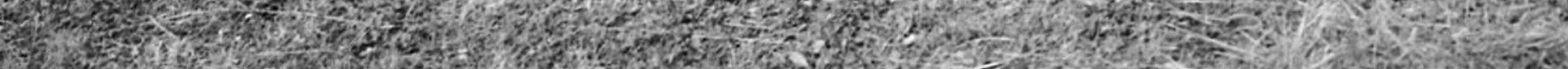




\title{
Quando il Moderno cerca radici. \\ Casa Balmelli di Tita Carloni e Luigi Camenisch
}

When the Modern puts down roots.

Casa Balmelli of Tita Carloni and Luigi Camenisch

\begin{abstract}
The article reconstructs the genesis of Casa Balmelli, designed and built in Rovio (Canton Ticino) in 1956-1957 by Tita Carloni and Luigi Camenisch. Among Carloni's first works in Ticino after the completion of his studies at the ETH Zurich, Casa Balmelli has often been presented as an example of the current of Organic Architecture that developed in Ticino in the 1950s and saw a particularly flourishing phase in the following decade. However, while the house certainly embodies organic features by aiming at a perfect integration with its surroundings through the use of natural materials and the geometric reinterpretation of the landforms of its setting, its internal spatial qualities have little to do, for instance, with Wrightian models. Rather, the ordering function attributed to geometry is its most notable quality and is a common denominator of the research conducted in Ticino on the organic architecture front: a geometry that relates to the characteristics of the site and seeks a certain degree of formal abstraction.
\end{abstract}

\section{Nicola Navone}

(Lugano, 1967) is vice director of the "Archivio del Moderno", professor at the Accademia di Architettura di Mendrisio - Università della Svizzera italiana, and a member of the Doctoral College "Architecture. Innovation and Heritage" - Università degli Studi Roma Tre.
Keywords

Cantone Ticino, Modernism, organicism, Organic Architecture, geometry, materials. 
Chi, valicata la frontiera di Chiasso, risale verso nord l'autostrada A2 e, superata Mendrisio, s'affaccia sul paesaggio del Ceresio, vedrà davanti a sé, a mezza costa del Monte Generoso, il villaggio di Rovio. O meglio: vedrà le villette e le case ad appartamenti cresciute lungo il declivio che culmina nel pianoro su cui sorge, dall'XI secolo, l'oratorio dedicato a San Vigilio. Per quanto sia acuta la sua vista, e anche ammettendo che si tratti del passeggero, libero di lasciar divagare a piacere il proprio sguardo, difficilmente percepirà il profilo dentato adagiato sul rilievo che corona il pianoro: il profilo, appunto, di casa Balmelli.

Progettata e costruita fra il 1956 e il 1957, questa piccola casa è tra le prime opere realizzate da Tita Carloni (1931-2012) dopo essere tornato in Ticino da Zurigo, dove aveva vagheggiato di stabilirsi insieme ad altri giovani colleghi ticinesi, una volta compiuti gli studi al Politecnico federale, se una serie di commesse non lo avesse richiamato nella terra na-

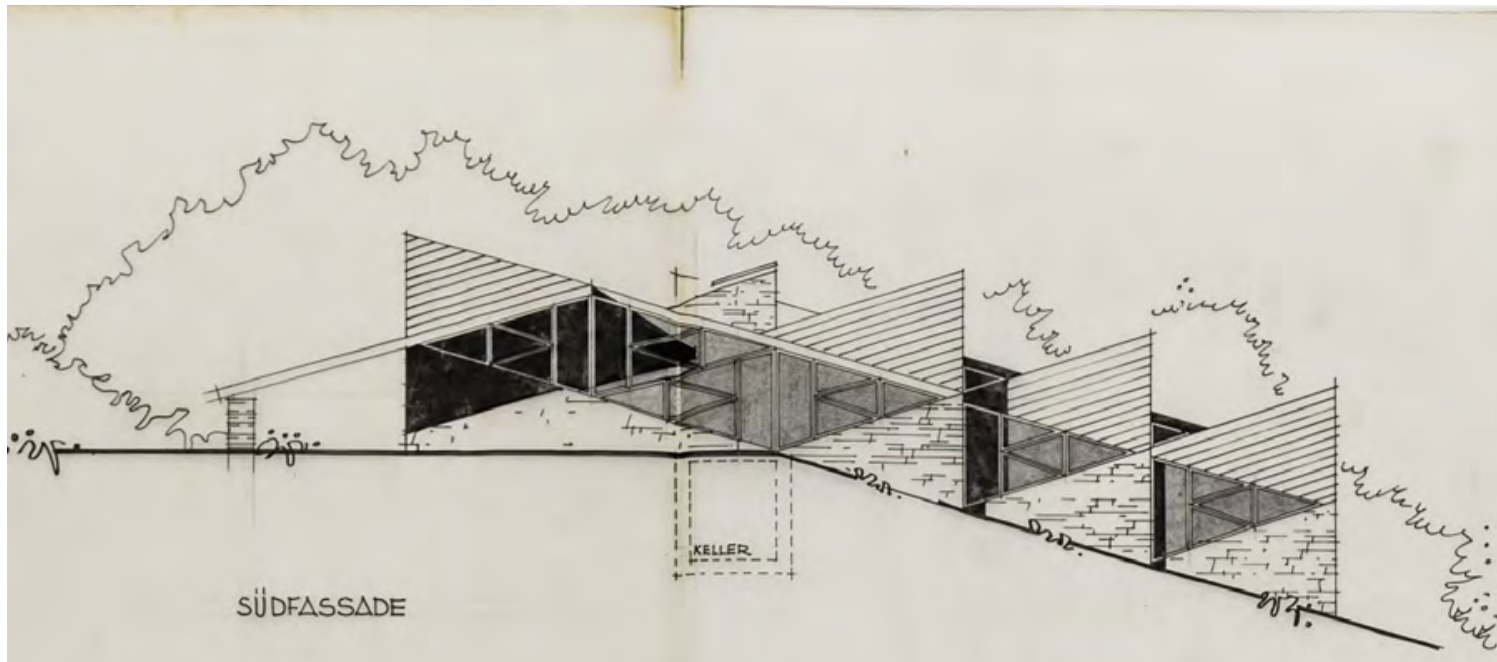

ZUM KELLER
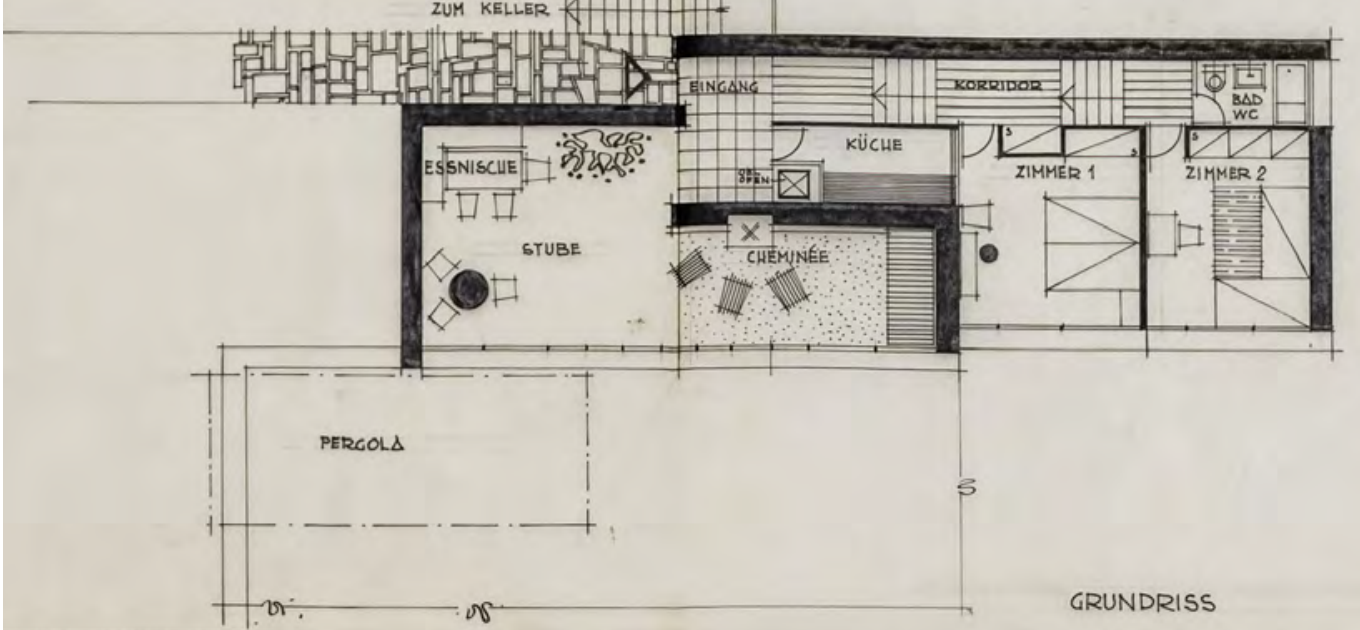
tia. Qui si era associato nel 1956 con Luigi Camenisch, un architetto maggiore di alcuni anni, con il quale aveva progettato delle case popolari per l'associazione "Pro Familia", a Lugano, e con il quale collaborerà sino al 1961, realizzando numerose altre opere fra le quali casa Balmelli (la cui prima variante documentata, risalente al 15 febbraio 1956, reca tuttavia la sola firma di Carloni).

Il committente, Hans Balmelli (1928-2019), era titolare di un'impresa di pittura fondata dal padre, nel 1924, a Zollikon, comune affacciato sul lago di Zurigo lungo la Goldküste, la riva orientata a solatio, luogo di residenza dell'alta borghesia zurighese. Di origini ticinesi, Giovanni era nato e cresciuto oltralpe, assimilandosi ai suoi concittadini sino a germanizzare il proprio nome e a ricorrere in-

Fig. 1 variabilmente al tedesco nella corrispondenza con Progetto di l'architetto.

massima; prospetto meridionale e pianta, planimetria, 15 febbraio 1956.
Balmelli delle trattative che lui e suo padre Taddeo (una personalità nota nel cantone, pittore-decoratore come Balmelli, docente e direttore della Scuola dei pittori a Lugano) avevano intavolato per trovare un terreno nei pressi dell'oratorio di San Vigilio, dove si poteva godere di una perfetta esposizione e della vista verso il lago, gli ultimi rilievi delle Prealpi e, in una lontananza brumosa, la pianura padana. La scelta era infine caduta, per la strenua resistenza del proprietario di un terreno a valle del pianoro, su cui si erano appuntate le attenzioni di architetto e committente, su un lotto di proprietà della stessa famiglia Carloni, situato sulla minuscola collina che emerge, come un ultimo sussulto geologico, dal pianoro di San Vigilio. Il 24 novembre 1955 Hans Balmelli esponeva all'architetto il programma della piccola casa di vacanza e gli orientamenti del proprio gusto: «Nach unseren Vorstellungen sollte das Haus ungefähr so aussehen: 1 riesengrosse Stube mit Cheminee / 2 Schlafzimmer / $1 \mathrm{Bad}$ / 1 Miniaturküche (damit die Frau nicht zu viel in der Küche ist). Innen soll es genial und modern eingerichtet sein, aussen muss es gut in die Landschaft passen (Tessinerstil). Wir verabscheuen die Deutschschweizer-Häuser, wie sie leider auch schon in Rovio entstanden sind» («Secondo i nostri intendimenti la casa dovrebbe presentarsi così: 1 grande Stube con camino / 2 camere da letto / 1 bagno / 1 minuscola cucina (così che mia moglie non stia troppo in cucina). All'interno dev'essere geniale e moderna, all'esterno deve integrarsi nel paesaggio (Stile ticinese). Detestiamo le case "alla svizzera tedesca" che, purtroppo, sono già sorte anche a Rovio»).

Carloni avrà sussultato leggendo la parola «Tessinerstil», benché preceduta e attenuata dalla coppia di aggettivi «genial und modern» (che avrà accolto con ironia): di fatto, si trovava confrontato con un incarico ricorrente nel Ticino degli anni Cinquanta, vale a dire la casa di vacanza per il più o meno facoltoso cliente d'Oltralpe (svizzero o tedesco che fosse) che intendeva godere del clima mite della "Sonnenstube der Schweiz". Quelle case, per l'appunto, che si stavano abbarbicando sui declivi delle Prealpi declinando in luoghi comuni ricorrenti un immaginario vernacolo ticinese, che poco o nulla aveva da spartire con la tradizione costruttiva locale (così come, in una realtà variegata e frammentata con il Cantone Ticino, non esisteva alcun «Tessinerstil»). Ma Carloni, benché giovane (24 anni da poco compiuti, quando riceve l'incarico da Balmelli), non era certo disposto a compromessi. Quella sarebbe stata anzi l'occasione per dimostrare che era possibile progettare un'architettura radicata nel tempo e nello spazio: vale a dire sinceramente moderna e al contempo in dialogo con le caratteristiche del sito e una specifica tradizione costruttiva. 
Osservando gli elaborati grafici conservati fra le carte dell'architetto, casa Balmelli pare nascere come Minerva dal capo di Giove: nessun abbozzo preliminare, nessuna esitazione nell'individuare l'impianto che la caratterizza. Come si è detto, la prima tavola di presentazione, stesa a china e munita di cartiglio, risale al 15 febbraio 1956. La giacitura della casa è perpendicolare alle curve di livello e i tre ambienti principali (l'ampia «Stube» e le due camere da letto) sono posti a quote diverse e raccordati da una lunga scala-corridoio, per adattarsi all'orografia e ridurre al minimo lo scavo nel terreno roccioso. La copertura è articolata in una sequenza di falde, simili a una sorta di "shed", che genera una tensione dinamica tra le diagonali delle falde e il profilo del terreno. La facciata principale (che in questa prima proposta presenta un leggero arretramento in corrispondenza delle camere) è rivolta a solatio e provvista di ampie aperture romboidali (nello spazio libero fra basamento e copertura), mentre quella opposta è completamente chiusa (salvo alcune feritoie che verranno aggiunte nel corso del progetto). L'uso di materiali locali (muri in pietra calcarea con intonaco «a rasapietra» - in analogia al vicino oratorio romanico - e coperture e serramenti in legno di castagno) avrebbe contribuito, come sostiene Carloni in una

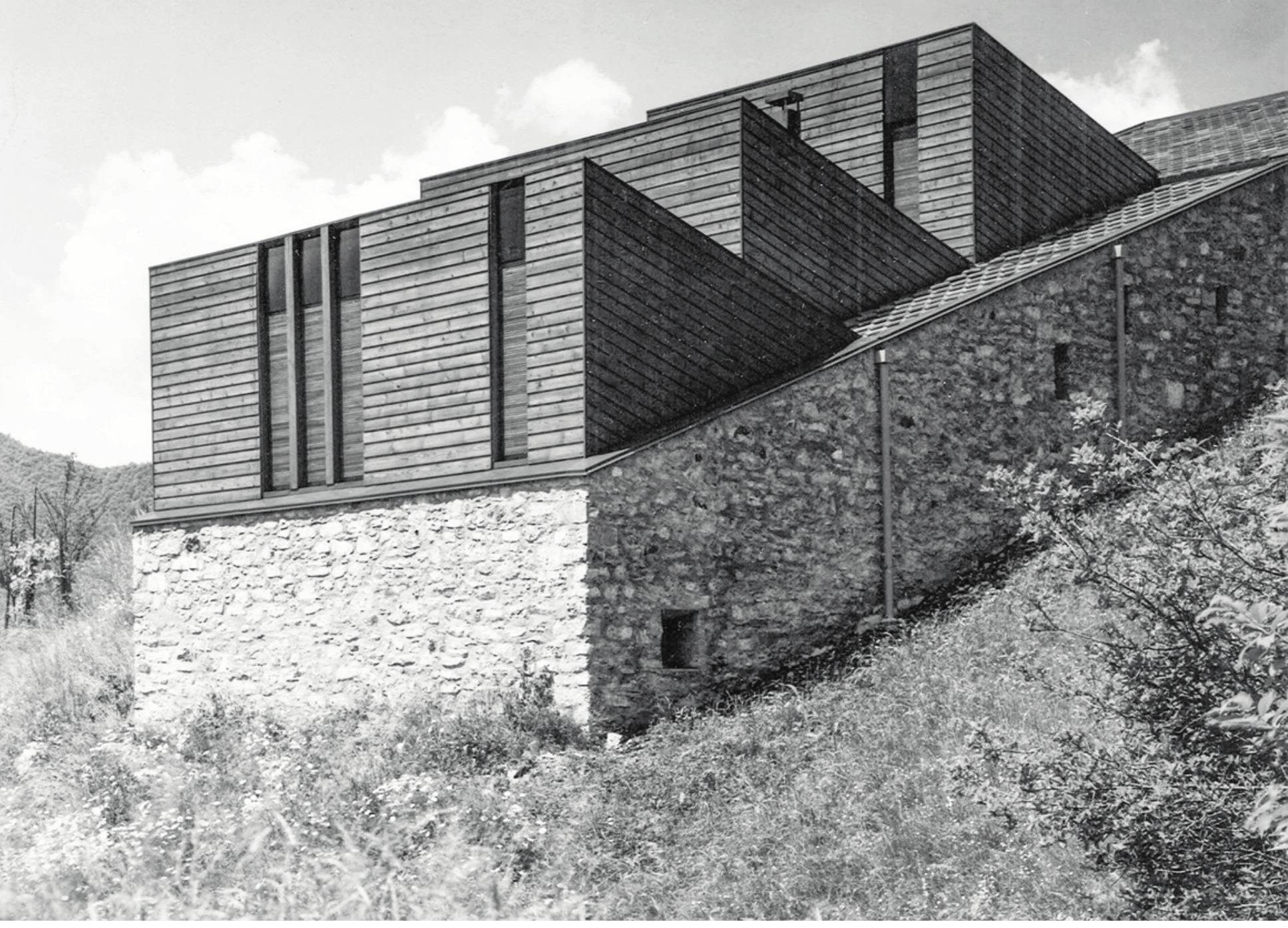


Fig. 2 ne dell'edificio nel paesaggio.

Vista da nord-est. La proposta, trasmessa al committente insieme ad alcune immagini del modello di studio, viene accolta con favore e da quel momento il progetto pro-

Fig. 3 Vista della "Stube".

Fig. 4

Progetto presentato per la licenza edilizia, piante, prospetti e sezioni 18 aprile 1956. cede rapidamente. Il 18 aprile 1956 Carloni e Camenisch firmano la tavola sinottica che sarà inviata alle autorità per il rilascio della licenza edilizia e poche settimane più tardi, nel maggio 1956, vengono allestiti gli esecutivi, che affinano ulteriormente il progetto e gli conferiscono l'assetto definitivo: i serramenti a trama diagonale hanno ora ceduto il campo a più semplici finestre verticali e le apertu- re praticate, nella variante intermedia, nella copertura a ponente, su richiesta della committenza sono portate a una quota inferiore e aperte nella muratura. Il cantiere prende avvio nell'estate di quello stesso anno, facendo capo a un'impresa e ad artigiani locali, e viene portato a termine entro la primavera del 1957. La cura dei dettagli e della loro esecuzione (evidente, ad esempio, nella qualità dell'apparecchio murario) è accompagnata dall'attenzione all'accordo cromatico dei prospetti, testimoniata dall'uso, affatto singolare, di tegole di colore verde, complementari alle opere di lattoneria tinteggiate di rosso «sangue di bue».

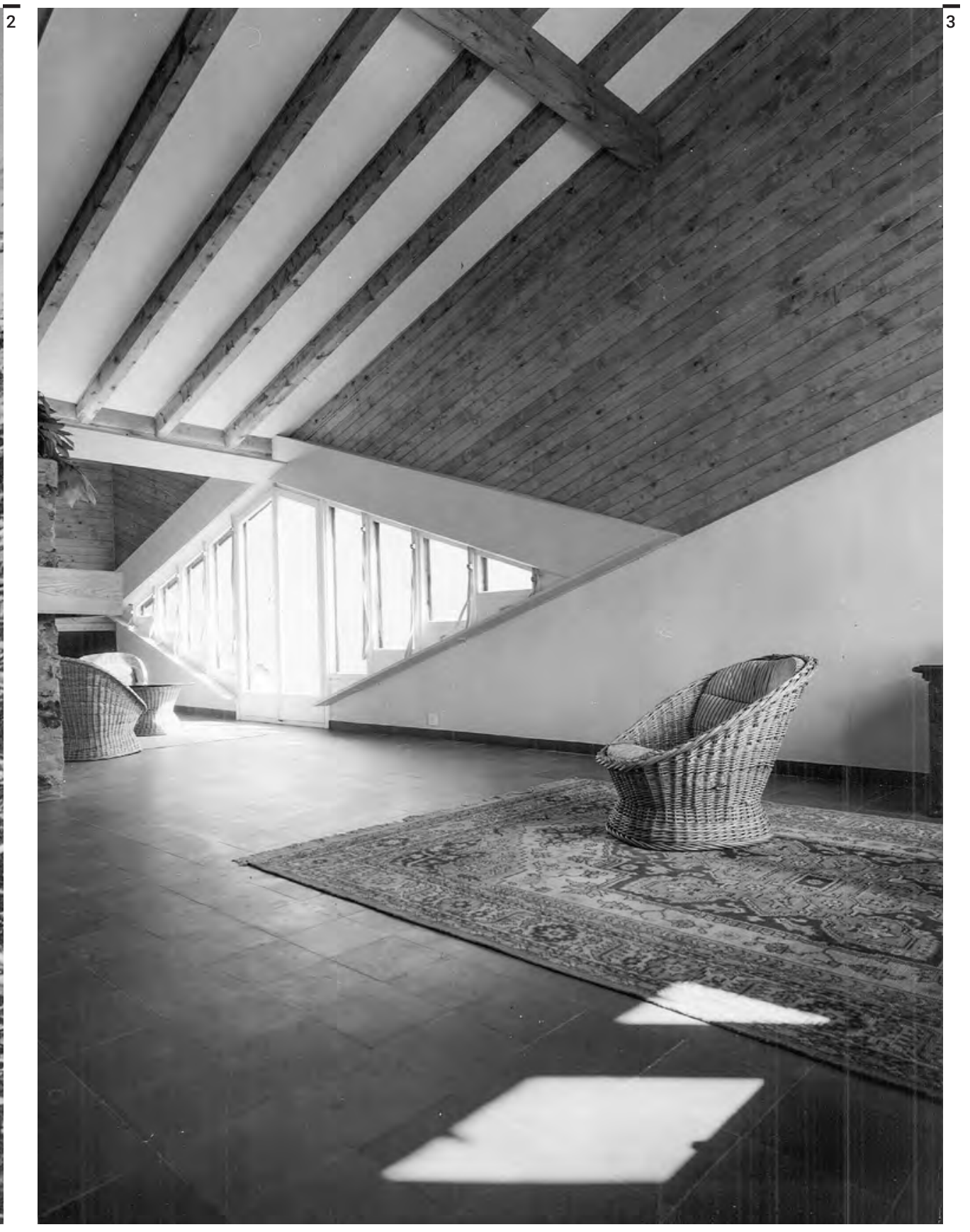




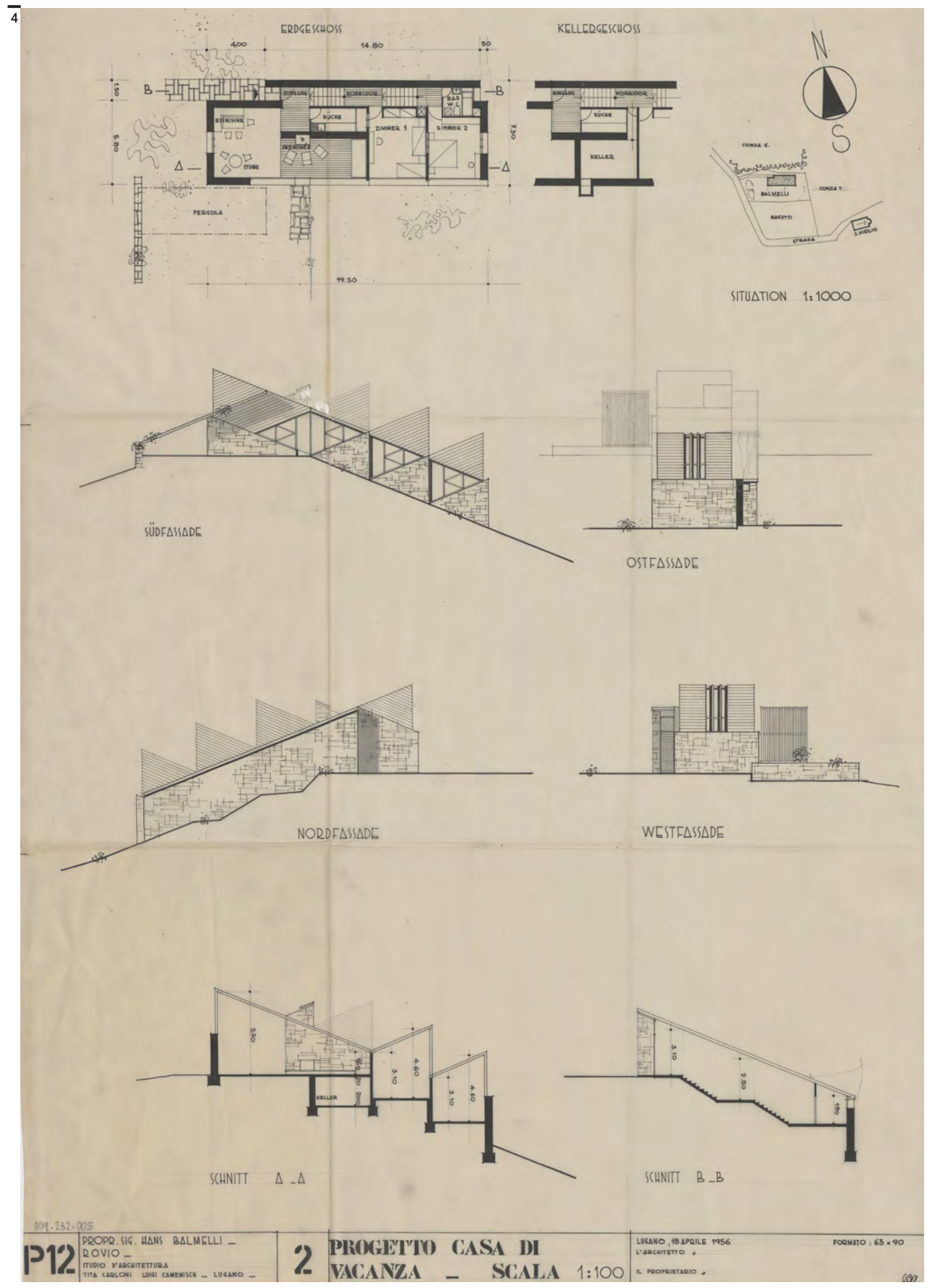


Fig. 5 Casa Balmelli suscita un immediato interesse non

Progetto esecutivo sezione lungo la scala-corridoio, 28 maggio 1956.

Fig. 6

Progetto esecutivo, prospetti, 19 maggio

1956. soltanto in Ticino, ma nel resto della Svizzera ( $A r$ chitecture: formes et fonctions, 1959; Werk, 1960), con qualche precoce risonanza europea (L'architettura: cronache e storia, 1960; Aujourd'hui: art et architecture, 1961). Su istigazione dello stesso Carloni, casa Balmelli verrà sovente portata ad esempio di quella corrente "organicista" che si sviluppa in Ticino negli anni Cinquanta e conosce un momento di par- ticolare fioritura nel decennio successivo: una corrente a cui vengono ascritti architetti come Franco Ponti, Peppo Brivio (piuttosto orientato, invece, a sviluppare una rigorosa grammatica personale, muovendo dal precoce interesse per la poetica neoplastica e per l'Arte Concreta), o lo stesso Rino Tami, per alcuni progetti della sua piena maturità. Tuttavia, se casa Balmelli assume certamente istanze organiciste quando mira a una perfetta integra-
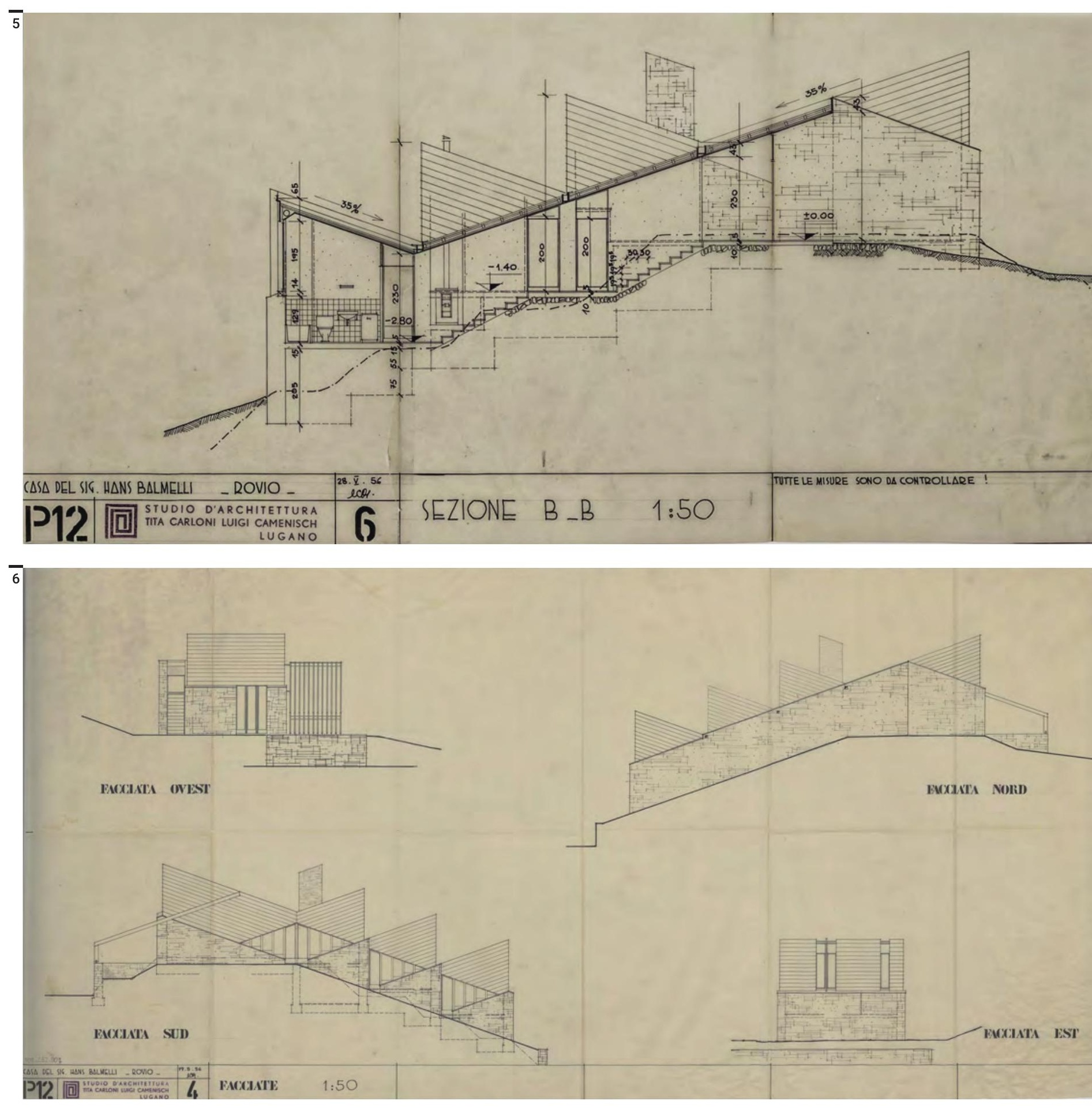
zione nel sito attraverso l'uso di materiali "naturali" e la reinterpretazione in chiave geometrica del contesto orografico, essa presenta una spazialità interna che poco ha a che vedere, ad esempio, con i modelli wrightiani (verso cui guarda, con coerenza e originalità, Franco Ponti) e le loro piante articolate, le transizioni spaziali fluide, la continuità fra interno ed esterno, a cui appare estranea la composizione paratattica e, in generale, l'articolazione planimetrica di casa Balmelli. Spicca, invece, la funzione ordinatrice attribuita alla geometria, che costituisce un denominatore comune delle ricerche compiute in Ticino sul versante organicista. Una geometria che si pone in relazione con le caratteristiche del sito e mira a un certo grado di astrazione formale. Senza dimenticare che la trama delle diagonali contribuisce ad alterare la percezione delle dimensioni e della configurazione dell'edificio, la cui immagine si contrae e si dilata secondo l'angolo da cui l'osserviamo, come per un surrettizio e ipotetico

Fig. 7 omaggio alle ricerche dell'arte cinetica e come ulteVista dall'oratorio di riore manifestazione della ricchezza e della forza di San Vigilio.

\section{Bibliografia}

Architecture: formes et fonctions (1959), vol. 6, pp. 154-155. Aujourd'hui: art et architecture (1961), vol. 5, pp. 66-67. Carloni Tita (1975), «Notizen zu einer Berufschronik», in Steinmann Martin, Boga Thomas (a cura di), Tendenzen. Neuere Architektur im Tessin, ETHZ Organisationsstelle für Austellungen des Institutes gta, Zürich, pp. 16-21 (nuova ed. Birkhäuser, Basel, 2010).

Carloni Tita (2011), «Tita Carloni, una voce critica dell'architettura ticinese. Intervista», in Archivio Storico Ticinese, seconda serie, n. 146, pp. 33-62

Fumagalli Paolo (1992), «L'architettura degli anni '50 nel Ticino: gli anni di "fondazione"», in I nostri monumenti storici, a. XLIII, n. 3, pp. 414-425.

L'architettura: cronache e storia (1960), vol. 58, p. 260.

Navone Nicola (2004), «Fonti, paradigmi, modelli: brevi note sull'architettura degli anni cinquanta in Ticino», in Archivio Storico Ticinese, seconda serie, n. 136, pp. 257-280.

Werk (1960), a. XLVII, n. 4, pp. 120-122.

\section{Fonti}

Fondazione Archivi Architetti Ticinesi, Fondo Tita Carloni, progetto 232, cartella 46 (per gli elaborati grafici), scatola 55 (per i documenti testuali).

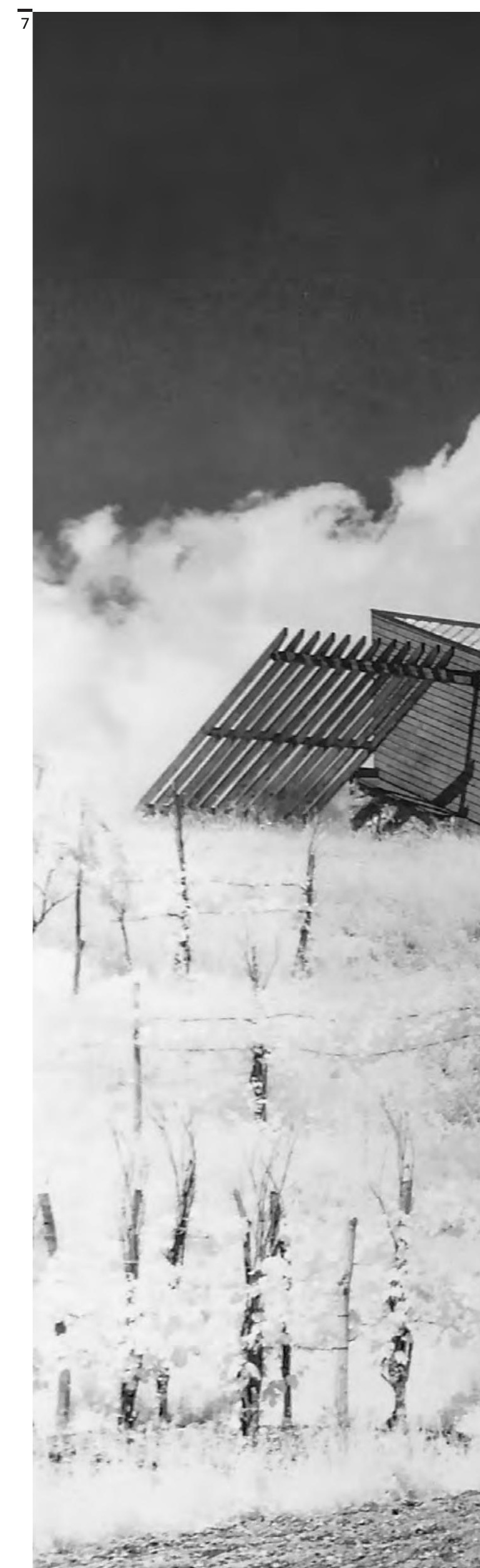




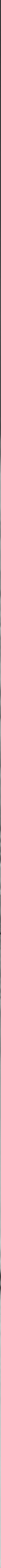

2. (1)

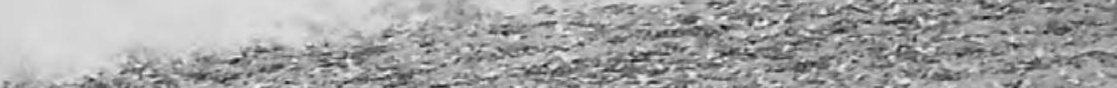

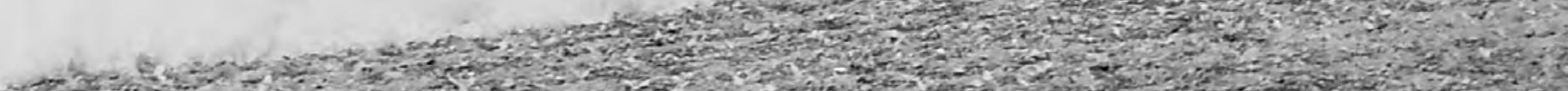

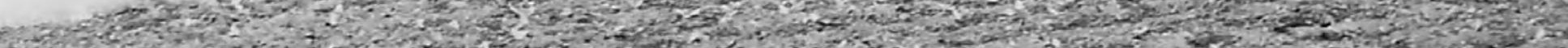

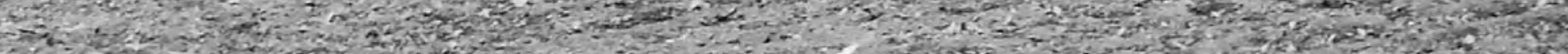
65 (5)

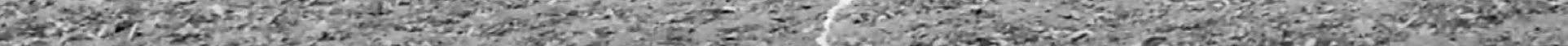

